\title{
ADJOINTS OF MULTIPOINT-INTEGRAL BOUNDARY VALUE PROBLEMS
}

R. C. BROWN AND ALLAN M. KRALL

Abstract. The dual system to $L y=y^{\prime}+P y$,

$$
\sum_{i=0}^{\infty} A_{i} y\left(t_{i}\right)+\int_{0}^{1} K(t) y(t) d t=0
$$

is found when the setting is $L_{n}^{p}(0,1), 1<p<\infty$.

Introduction. Let $X$ denote the Banach space $L_{n}^{p}(0,1)$, consisting of $n$-dimensional vectors under the norm

$$
\|x\|=\left[\int_{0}^{1}\left[\sum_{i=1}^{n}\left|x_{i}(t)\right|^{2}\right]^{p / 2} d t\right]^{1 / p}
$$

$1<p<\infty$. In $X$ and $X^{*}\left(=L_{n}^{q}(0,1), 1 / p+1 / q=1\right)$ let us define the following subspaces:

(a) Let $D^{\prime}$ denote those vectors in $X$ which are absolutely continuous.

(b) Let $D_{0}^{+}$denote those vectors in $X^{*}$ which are absolutely continuous and vanish at $t=0, t=1$.

(c) Let $A_{i}, i=0, \ldots$, be $m \times n$ constant matrices satisfying $\sum_{i=0}^{\infty}\left\|A_{i}\right\|<$ $\infty\left(\|\cdot\|\right.$ here denotes a convenient norm) and $\bigcap_{i=0}^{\infty} \operatorname{ker} A_{i}^{*}=0$. Let $\left\{t_{i}\right\}_{i=0}^{\infty}$ be a collection of points in $[0,1]\left(t_{0}=0, t_{1}=1\right)$, and let $K(t)$ be an $m \times n$ matrix valued function whose rows are in $X^{*}$. For all $y$ in $D^{\prime}$ we define the (discontinuous) boundary functional $V$ by

$$
V y=\sum_{i=0}^{\infty} A_{i} y\left(t_{i}\right)+\int_{0}^{1} K(t) y(t) d t,
$$

and denote by $D$ the kernel of this functional. $V y=0$ for all $y \in D$.

(d) Let $D^{+}$denote those vectors $z$ in $X^{*}$ for which there exists an $m \times 1$ matrix valued functional $\phi(z)$ such that

(1) $z(t)+\sum_{i=0}^{\infty} A_{i}^{*} \phi(z) \lambda\left(t_{i}, 1\right]$ is absolutely continuous on $[0,1]$. $\left(\lambda\left(t_{i}, 1\right]\right.$ is the characteristic function of $\left(t_{i}, 1\right]$.)

Presented to the Society, January 18, 1972; received by the editors November 1 , 1971 and, in revised form, March 20, 1972.

AMS (MOS) subject classifications (1970). Primary 34B05, 34B10, 34B25; Secondary 44A60.

(c) American Mathematical Society 1973 
(2) If $P(t)$ is a continuous $n \times n$ matrix valued function, then the expression $l^{+} z=-z^{\prime}+P^{*} z+K^{*} \phi(z)$ exists a.e. and is in $X^{*}$.

We note that $\phi$ is uniquely defined, for if two such $\phi, \phi_{1}$ and $\phi_{2}$, exist, then by subtraction $\sum_{i=0}^{\infty} A_{i}^{*}\left(\phi_{1}-\phi_{2}\right) \lambda\left(t_{i}, 1\right]$ would be absolutely continuous. This is impossible. Further note that the range of $\phi$ is all of $R^{m}$, that $D_{0}^{+} \subset D^{+}$, and that $\phi(z)=0$ if and only if $z \in D_{0}^{+}$. Hence $D^{+}$is nonempty.

Finally, letting $l y=y^{\prime}+P y, l^{+} z$ as given above, we define the following operators:

(a) $L^{\prime}$ is given by $L^{\prime} y=l y$ for all $y$ in $D^{\prime}$.

(b) $L_{0}^{+}$is given by $L_{0}^{+} z=l^{+} z$ for all $z$ in $D_{0}^{+}$.

(c) $L$ is given by $L y=l y$ for all $y$ in $D$.

(d) $L^{+}$is given by $L^{+} z=l^{+} z$ a.e. for all $z$ in $D^{+}$.

Our principal result is that $L$ and $L^{+}$are dual operators. Brown [1] has previously shown that $D$ is dense in $X$, so $L^{*}$ exists. Further Bryan [2] used $L^{+}$as the basis of a definition of $L^{*}$. The result is well known under the classical endpoint conditions. However, because of the possible density of the boundary points $\left\{t_{i}\right\}$ in the interval $[0,1]$, the techniques now used are different from those used previously.

The result also generalizes similar results recently obtained by Green and Krall [4] and Krall [5].

The closure of $L^{+}$.

THEOREM 1. The operator $L^{+}$, defined by $L^{+} z=-z^{\prime}+P^{*} z+K^{*} \phi(z)$, for all $z \in D^{+}$, is closed.

ProOF. We recall that the operator $L^{+}$is closed if when $\lim _{k \rightarrow \infty} z_{k}=$ $z\left(z_{k} \in D^{+}\right)$, and $\lim _{k \rightarrow \infty} L^{+} z_{k}=y$, then $z \in D^{+}$, and $L^{+} z=y$.

Let $\varepsilon(s)=\sum_{i=0}^{\infty} A_{i}^{*} \lambda\left(t_{i}, 1\right](s)$. Then

$$
\begin{aligned}
z_{k}(s)-z_{l}(s)= & \int_{0}^{s}\left(z_{k}^{\prime}-z_{l}^{\prime}\right) d \xi+\varepsilon(s) \phi\left(z_{l}-z_{k}\right) \\
= & \int_{0}^{s} L^{+}\left(z_{l}-z_{k}\right) d \xi+\int_{0}^{s} P^{*}\left(z_{k}-z_{l}\right) d \xi \\
& +\left[\int_{0}^{s} K^{*} d \xi-\varepsilon(s)\right] \phi\left(z_{k}-z_{l}\right) .
\end{aligned}
$$

Since

and

$$
\left\|\int_{0}^{s} L^{+}\left(z_{k}-z_{l}\right) d \xi\right\|_{X^{*}} \leqq\left\|L^{+}\left(z_{l}-z_{k}\right)\right\|_{X^{*}}
$$

$$
\left\|\int_{0}^{s} P^{*}\left(z_{k}-z_{l}\right) d \xi\right\|_{X^{*}} \leqq\left\|P^{*}\right\|\left\|z_{k}-z_{l}\right\|_{X^{*}}
$$


by Hölder's inequality, we have

$$
\begin{aligned}
&\left\|\left[\int_{0}^{s} K^{*} d \xi-\varepsilon(s)\right] \phi\left(z_{k}-z_{l}\right)\right\|_{X^{*}} \\
& \leqq\left\|z_{k}-z_{l}\right\|_{X^{*}}+\left\|P^{*}\right\|\left\|z_{k}-z_{l}\right\|_{X^{*}}+\left\|L^{+}\left(z_{l}-z_{k}\right)\right\|_{X^{*}}
\end{aligned}
$$

By assumption, each of the terms on the right approaches 0 as $k, l$ approach $\infty$. Therefore the functions

$$
F_{k}(s)=\left[\int_{0}^{s} K^{*} d \xi-\varepsilon(s)\right] \phi\left(z_{k}\right)
$$

converge in measure. Hence there is a subsequence $F_{k_{j}}(s)$ which converges almost everywhere. Letting $s$ approach $t_{i}$ from above and below implies $F_{k_{j}}\left(t_{i}^{+}\right)-F_{k_{j}}\left(t_{i}^{-}\right)=A_{i}^{*} \phi_{k_{j}}$ converges. Therefore $\phi_{k_{j}}-\phi_{k_{l}}{ }^{1}$ approaches the kernel of $A_{i}^{*}$ as $k_{j}, k_{l}$ approach $\infty$. Since this holds for all $i$, and $\bigcap_{i=0}^{\infty} \operatorname{ker} A_{i}^{*}=0$, we conclude that $\phi_{k_{j}}-\phi_{k_{l}}$ approaches 0 . In other words $\phi_{k_{j}}$ converges.

Since

$$
z_{k_{j}}(s)=-\int_{0}^{s} L^{+} z_{k_{j}} d \xi+\int_{0}^{s} P^{*} z_{k j} d \xi+\left[\int_{0}^{s} K^{*} d \xi-\varepsilon(s)\right] \phi\left(z_{k_{j}}\right)
$$

and $\phi\left(z_{k_{j}}\right)$ converges, we may take limits to find

$$
z(s)=-\int_{0}^{s} y d \xi+\int_{0}^{s} P^{*} z d \xi+\left[\int_{0}^{s} K^{*} d \xi-\varepsilon(s)\right] \phi
$$

or

$$
z(s)+\varepsilon(s) \phi=-\int_{0}^{s} y d \xi+\int_{0}^{s} P^{*} z d \xi+\int_{0}^{s} K^{*} d \xi \phi .
$$

Since the right is absolutely continuous, $z$ satisfies the first requirement of elements in $D^{+}$. Differentiating, we find $z^{\prime}=-y+P^{*} z+K^{*} \phi$, or $y=l^{+} z$ a.e., the second condition.

The duals of $L$ and $L^{+}$. Let us denote the dual of an operator $A$ by $A^{*}$.

LEMMA. $\left(L_{0}^{+}\right)^{*}=L^{\prime}$.

Proof. This is well known. See Goldberg [3, p. 51].

THEOREM 2. $\left(L^{+}\right)^{*}=L$.

${ }^{1}$ The matrix $A_{i}^{*}$ defines an isomorphism $f_{i}$ from the factor space $R^{m} / \operatorname{ker} A_{i}^{*}$ onto the range of $A_{i}^{*}$. Thus $A_{i}^{*} \phi_{k}$ approaches 0 if and only if $\phi_{k}+\operatorname{ker} A_{i}^{*}$ approaches ker $A_{i}^{*}$. 
Proof. Trivial modifications of a computation found in Green and Krall [4] show that if $y \in D$ and $z \in D^{+}$, then

Thus $L \subset\left(L^{+}\right)^{*}$.

$$
\begin{aligned}
(L y, z)-\left(y, L^{+} z\right) & =\int_{0}^{1}\left[z^{*}(L y)-\left(L^{+} z\right)^{*} y\right] d s \\
& =-\phi^{*}(z)\left[\sum_{i=0}^{\infty} A_{i} y\left(t_{i}\right)+\int_{0}^{1} K y d s\right]=0 .
\end{aligned}
$$

To show the reverse inclusion, let $z \in D_{0}^{+}$. Then, since $D_{0}^{+} \subset D^{+}$and $\phi(z)=0$, we find $L_{0}^{+} \subset L^{+}$. This implies $\left(L^{+}\right)^{*} \subset\left(L_{0}^{+}\right)^{*}=L^{\prime}$. Hence the domain of $\left(L^{+}\right)^{*}$ is contained in $D$.

For arbitrary $z \in D^{+}$, the calculation of the first part of the proof shows

$$
\phi^{*}(z)\left[\sum_{i=0}^{\infty} A_{i} y\left(t_{i}\right)+\int_{0}^{1} K y d s\right]=0 .
$$

Since the range of $\phi$ is $R^{m}$, it is the term in brackets which vanishes. Thus the domain of $\left(L^{+}\right)^{*}$ satisfies the boundary condition and is in $D$. Therefore $\left(L^{+}\right) * \subset L$, and the two are equal.

THEOREM 3. $L^{*}=L^{+}$.

Proof. Since $L^{+}$is closed by Theorem $1,\left(L^{+}\right)^{* *}=L^{+}$. But since $\left(L^{+}\right)^{*}=L$ by Theorem 2 , we find $L^{*}=\left(L^{+}\right)^{* *}=L^{+}$.

\section{REFERENCES}

1. R. C. Brown, The existence of the adjoint in linear differential systems with discontinuous boundary conditions, Ann. Mat. Pura Appl. (to appear).

2. R. N. Bryan, A linear differential system with general linear boundary conditions, J. Differential Equations 5 (1969), 38-48. MR 38 \#1312.

3. S. Goldberg, Unbounded linear operators: Theory and applications, McGraw-Hill, New York, 1966. MR 34 \#580.

4. G. B. Green and A. M. Krall, Linear differential systems with infinitely many boundary points, Ann. Mat. Pura Appl. 91 (1972), 53-67.

5. A. M. Krall, Differential-boundary operators, Trans. Amer. Math. Soc. 154 (1971), 429-458. MR 42 \#6328.

Department of Mathematics, Pennsylvania State University, University Park, PenNSYlVANia 16802

Current address (R. C. Brown): Mathematics Research Center, University of Wisconsin, Madison, Wisconsin 53706 